\title{
Strategic plan of the Canadian Institutes of Health Research Institute of Nutrition, Metabolism, and Diabetes
}

\author{
Philip M Sherman MD FRCPC, Mary-Jo Makarchuk MSc MHSc RD, Paul Belanger BScN, Eve A Roberts MD FRCPC
}

PM Sherman, M-J Makarchuk, P Belanger, EA Roberts. Strategic plan of the Canadian Institutes of Health Research Institute of Nutrition, Metabolism, and Diabetes. Can J Gastroenterol 2011;25(10):560-564.

The present document provides the new and updated strategic plan for the Institute of Nutrition, Metabolism, and Diabetes (INMD) of the Canadian Institutes of Health Research. This plan provides an overarching map for the strategic activities of the INMD during the five years from 2010 to 2014 . These strategic priorities will guide the way that the INMD uses its resources over this period of time, and will provide opportunities to build new partnerships and strategic alliances that enhance and leverage the capacity to fund targeted research initiatives.

Key Words: Gastroenterology; Health policy; Hepatology research; Strategic plan

The mandate of the Canadian Institutes of Health Research 1 (CIHR) Institute of Nutrition, Metabolism, and Diabetes (INMD) is broad, and covers a range of conditions and problems affecting the endocrine system, the digestive system, the kidneys and the liver. Diet has been associated with many chronic diseases that contribute significantly to the economic burden of disease in Canada. Chronic conditions that fall within the INMD mandate are among the most costly to Canadians, in terms of both economic and social impacts.

Excellence in health research is the raison d'être of the CIHR. This new strategic plan provides an opportunity for the INMD to build on what was accomplished in the past by supporting Canadian research excellence in the areas of obesity and healthy body weight, while moving forward to tackle some of the greatest health challenges facing Canadians today. The new priorities also position the INMD to contribute to CIHR's overall success in achieving the strategic directions established in the CIHR's recently released strategic plan, Health Research Roadmap: Creating innovative research for better health and health care.

Being strategic enables the CIHR institutes to focus on specific research priorities, which ideally results in building research capacity while creating new knowledge and translating it to improving the health and quality of life of Canadians. In sharpening the INMD focus, there are risks of alienating some stakeholders because they may feel disenfranchised if they are unable to identify their particular research interests within the stated research priorities. By increasing the number of strategic INMD research priorities from one to four, the INMD is sending a clear message to the research communities that it will be inclusive and sensitive to their needs.

The INMD is committed to evaluating the impact of targeted research funding that is supported when implementing this strategic plan. The Institute Advisory Board (IAB) will meet regularly to discuss progress, make certain that the implementation of the updated strategic plan is on track and ensure that the INMD responds appropriately to emerging needs. The INMD will also meet with potential partners to discuss opportunities for collaboration in the implementation of this new strategic plan. The new strategic priorities will ensure

\author{
Le plan stratégique de l'Institut de la nutrition, du \\ métabolisme et du diabète des Instituts de \\ recherche en santé du Canada
}

Le présent document présente le plan stratégique nouveau et mis à jour de l'Institut de la nutrition, du métabolisme et du diabète (INMD) des Instituts de recherche en santé du Canada. Ce plan fournit un cadre déterminant pour les activités stratégiques de l'INMD pendant les cinq années qui commencent en 2010 et se terminent en 2014. Ces priorités stratégiques orienteront la manière dont l'INMD utilisera ses ressources pendant cette période, et fourniront des occasions de nouer de nouveaux partenariats et de nouvelles alliances stratégiques qui amélioreront et exploiteront la capacité de financer des projets de recherche ciblés. that the INMD continues to make a strong contribution to the CIHR in achieving its goal of improving the health of all Canadians.

\section{CIHR}

The CIHR is the major federal agency responsible for funding health research in Canada. Created under the CIHR Act, which came into force in June 2000, it comprises 13 virtual institutes that are mandated to support health research in four major areas: Pillar 1 - biomedical; Pillar 2 - clinical; Pillar 3 - health systems and services; and Pillar 4 - population and public health.

Each institute is headed by a scientific director who receives guidance and advice from its IAB. IABs are comprised of national and international representatives of the public, private and nonprofit sectors including the research community and health practitioners. IAB membership is balanced geographically across the pillars and by sex, and is inclusive of different stages of career development. The institutes are formally accountable to both the president and governing council of the CIHR and, through the Minister of Health, to Parliament.

CIHR was created to transform health research in Canada by funding more research in targeted priority areas, building research capacity in underdeveloped areas, such as population health and health services research, training the next generation of health researchers and focusing on knowledge translation so that the results of research are transformed into policies, practices, procedures, products and services.

CIHR's mandate is to excel, according to internationally accepted standards of scientific excellence, in the creation of new knowledge and its translation into improved health for Canadians, more effective health services and products, and a strengthened Canadian health care system.

To assure Canadians that their investments in health research are used wisely, CIHR embraces values that permeate all aspects of the organization's activities and relationships with others. These core values provide critical context for the new INMD Strategic Plan and are listed in Table 1.

\footnotetext{
Canadian Institutes of Health Research, Ottawa, Ontario

Correspondence: Dr Philip M Sherman, Institute of Nutrition, Metabolism, and Diabetes, Room 207L, Banting Building, 100 College Street, Toronto,

Ontario M5G 1L5. Telephone 416-978-1315, fax 416-978-1334, e-mail philip.sherman@sickkids.ca

Received for publication April 7, 2011. Accepted April 11, 2011
} 
In October 2009, CIHR launched its new strategic plan, Health Research Roadmap: Creating innovative research for better health and health care. This new strategic plan lays out CIHR's commitment to pursue the following strategic directions over the next five years:

A. Invest in world class excellence;

B. Address health and health system research priorities;

C. Accelerate the capture of health and economic benefits of health research; and

D. Achieve organizational excellence, foster ethics and demonstrate impact.

\section{INMD}

The mandate of the INMD is to support research to enhance health in relation to diet, digestion, excretion and metabolism, and to address causes, prevention, screening, diagnosis, treatment, support systems, and palliation for a wide range of conditions and problems associated with hormone, digestive system, kidney and liver function. INMD's vision is to position Canada as a leader in knowledge creation through health research in relation to diet, digestion, excretion and metabolism that benefits all Canadians and the global community.

Unlike the other 12 institutes within the CIHR, INMD has pursued a single strategic focus since its inception. INMD's first strategic plan, released in 2002, outlined the initial goals and objectives for INMD's strategic focus on obesity and healthy body weight. In 2005, a mid-term evaluation of INMD identified that the selection of a single strategic focus was unique among the CIHR institutes and, although most stakeholders supported the decision to focus on this priority, some acknowledged that certain communities may have felt disenfranchised by this choice.

INMD's focus on obesity and healthy body weight as a single priority since 2002 was innovative. The focus resulted in growth in obesityrelated research capacity. Data from a bibliometric analysis commissioned by the INMD indicated that the number of peerreviewed articles published by CIHR-funded researchers increased dramatically from 1998 to 2007.

In 2008, the INMD IAB embarked on a process to identify future directions and strategic research priorities by undertaking an environmental scan. While there was a range of opinions offered by survey respondents with respect to retaining or changing the INMD strategic priority, the majority of respondents indicated that INMD should keep obesity and healthy body weight as a priority, but expand to include other priorities.

\section{METHODOLOGY}

INMD 2008 environmental scans

In July 2008, the INMD conducted an online survey of researchers and other key stakeholders as one component of an environmental scan on research and knowledge translation priorities. In that survey, respondents $(n=645)$ chose from among eight thematic areas and responded to a common series of questions in each area. The results of this environmental scan indicated that stakeholders perceived obesity and healthy body weight to be of continued importance; however, respondents recommended that INMD broaden its strategic focus.

In preparation for strategic planning, INMD commissioned a study to provide a scan of investment priorities of related funders. This scan provided a high-level overview of strategic research investment priorities, activities and future opportunities for research investment identified by a wide range of national and international organizations operating within a scope similar to the mandate areas of INMD. Because the scan was limited to publicly available data, it may not have been able to provide a complete picture of the funding landscape.

\section{Consultation with stakeholder groups}

From May to October 2009, the INMD team consulted a variety of stakeholders in INMD strategic planning summits. To maximize opportunities to meet with research communities and other stakeholders

\section{TABLE 1}

Core values of the Canadian Institutes of Health Research (CIHR) used in developing the strategic plan for the Institute of Nutrition, Metabolism, and Diabetes

Excellence: In all aspects of its work including research, knowledge translation and organizational services, CIHR strives to meet the highest international standards of excellence

Scientific integrity and ethics: CIHR upholds and promotes adherence to relevant research and organizational principles with utmost honesty, probity and professionalism. CIHR believes that excellent research, knowledge application and good governance require the development and application of sound ethical principles and processes

Collaboration: CIHR promotes, encourages and values collaboration among researchers in Canada and internationally. CIHR engages, collaborates and coordinates research activities with federal and provincial/territorial governments and departments, and nonprofit and private-sector organizations. CIHR is committed to openness, responsibility, fairness and mutual respect with all its researchers and partners. CIHR cannot fulfill its mandate without the collaboration and support of domestic and international partners

Innovation: CIHR values new ideas and creative approaches to addressing health and health system challenges in Canada and worldwide

Public interest: The public interest is of paramount importance in the creation and use of health knowledge through all research and related activities supported by the CIHR

while being conscious about operating expenses, INMD specifically sought opportunities to meet with stakeholders at existing conferences and scientific meetings where groups were already gathering. These meetings included the following: The Canadian Society for Nutritional Sciences/Canadian Society for Clinical Nutrition; Digestive Disease Week; The Canadian Public Health Association Conference; The Canadian Lipoprotein Conference; The Canadian Society of Endocrinology and Metabolism; World Diabetes Congress/ International Diabetes Federation; and The Obesity Society.

INMD also met with several stakeholders through smaller meetings and consultations, which included The Federal/Provincial/Territorial (F/P/T) Group on Nutrition; The Expert Group on Chronic Disease Prevention and Control (F/P/T); The Federal health portfolio (Health Canada, the Public Health Agency of Canada, and the First Nations and Inuit Health Branch) - Nutrition; and The Executive Board Meeting of the Canadian Society of Nephrology.

In total, approximately 200 stakeholders participated in either a strategic planning summit or consultation meeting in person. These stakeholders included investigators from across Canada, representing the breadth of the INMD mandate, as well as representatives from governments, professional associations and voluntary health organizations. In addition, INMD conducted an online survey of partner voluntary health organizations.

The IAB met in November, 2009, at the University of Manitoba (Winnipeg, Manitoba) to determine the new INMD strategic priorities. The first task was to develop criteria for decision making. These criteria evolved from input received from stakeholders who participated in INMD strategic planning summits, consideration of criteria used by other CIHR institutes, and reflection on the new CIHR strategic plan. The IAB used the criteria listed in Tables 2, 3 and 4 when selecting research priorities.

Members of the IAB unanimously agreed on the four new INMD strategic priorities. After the IAB meeting, the draft strategic priorities were sent out electronically to CIHR researchers identified through the CIHR database for their feedback. These researchers had applied to CIHR in the past for funding and identified their project(s) as relevant to INMD. Approximately 200 researchers responded to this web-based survey, the majority indicating that they either supported or strongly supported all four priorities. However, stakeholders also 


\section{TABLE 2}

Criteria employed for selecting the Institute of Nutrition, Metabolism, and Diabetes (INMD) research priorities

Addresses an identified knowledge gap
Likely to result in measurable results consistent with the Health Impact
Assessment Framework (ie, advance knowledge, build capacity, inform
decision-making, improve health and the health system, contribute to the
economy)
Likely to translate into affordable and sustainable interventions to improve
the health of Canadians and the Canadian health care system
Likely to effectively reduce the burden of illness/disease
Builds on Canadian research strength
Supports innovation, risk-taking and creativity in contributing to CIHR and
INMD mandates
Likely to secure Canada's place on the global stage in addressing health
challenges relevant to the INMD mandate

CIHR Canadian Institutes of Health Research

\section{TABLE 3}

Institute of Nutrition, Metabolism, and Diabetes (INMD) strategic research priorities were built on foundational principles

Research excellence through partnerships: INMD will engage, collaborate and coordinate partnerships at the national and international levels. We will leverage partnership opportunities with other CIHR institutes, government partners, voluntary health organizations, private sector partners and international partners to advance our strategic research priorities

Ethics: INMD adheres to CIHR's belief that excellent research, knowledge application, and good governance require the development of sound ethical principles and process. INMD will increase awareness of the ethical issues inherent in research relevant to our mandate, and will support a broad range of ethics research related to the strategic research priorities

Capacity: INMD will strive to provide the necessary support to attract and sustain talented researchers to advance the INMD strategic research priorities, in collaboration with our many partners

Knowledge translation: INMD will contribute to CIHR's role in knowledge translation by promoting the dissemination and application of new knowledge generated by INMD's strategic priorities

Evaluation: INMD will contribute to CIHR's overall strategic priority of demonstrating impact by monitoring and evaluating the impact of our efforts in advancing the strategic research priorities, building on the impact assessment framework adopted by CIHR

CIHR Canadian Institutes of Health Research

indicated that some of the priorities needed to be clarified. The results of the survey were used to fine-tune the draft INMD strategic priorities and make them more specific.

\section{RESULTS}

Strategic priority 1: Food and health

INMD aims to develop a stronger evidence base to inform future nutritional practice and food policy. INMD will foster research on the total diet and specific nutrients to enhance health and reduce the risk of chronic disease. This includes, for example, the evaluation of biomarkers of nutritional adequacy, emerging innovations in food engineering and the ethical issues posed by these changes, particularly with respect to people with vulnerabilities.

Rationale: There is a growing interest among many health and diseasebased organizations on the role of food and nutrition, nutrients, dietary components, and dietary patterns in preventing and controlling premature morbidity and mortality resulting from chronic disease $(1,2)$. It has been said that, "Chronic non-communicable diseases constitute the major burdens of illness and disability in almost all
TABLE 4

Factors that were considered in the development of the Institute of Nutrition, Metabolsm, and Diabetes (INMD) strategic plan

Transparency

Engagement of key stakeholders and research communities

Consistency and equity in engaging communities

Positioning INMD to support the CIHR mandate

Building links between INMD and other institutes

Building on accomplishments of INMD to date

CIHR Canadian Institutes of Health Research

countries of the world. They must urgently receive more resources, research and attention, as mapped out in these grand challenges. Inaction is costing millions of premature deaths throughout the world" (3). In Canada, chronic diseases, including diabetes and its complications, digestive diseases, cardiovascular disease and cancers are responsible for a significant portion of the burden of disease.

During the past decade, the amount of scientific literature related to food and nutrition and chronic disease expanded tremendously. However, there remains many unanswered research questions regarding food and health. In almost all cases, these questions require investigation from a variety of approaches. These include, but are not limited to, the basic physiological, metabolic and homeostatic mechanisms (eg, lymphatic growth response to salt intake, lipid droplet formation); clinical research related to food and nutrition, nutrient requirements and maintenance of health (eg, micronutrient interventional research); and food and nutrition policy research at the population level (eg, sodium reduction, food fortification, functional foods and food security). The overarching aim of this strategic priority is to foster research on food and health that results in improved nutritional status at the population level, compressed morbidity in relation to chronic disease, and evidence-informed policies and practice.

Strategic priority 2: Environments, genes and chronic disease INMD recognizes the influence of genes and the environment on the development of chronic disease. INMD will promote the acquisition of knowledge on the phenotypic variation of both complex and rare diseases, interactions with the human microbiome, and the health consequences of changes in the natural and built environments.

Rationale: The mandate of INMD includes both extremely rare and highly prevalent conditions affecting the gut, the genitourinary system, the liver and the endocrine system. The impact on the quality of life for affected Canadians is profound, and the resulting costs to both the health care system and the Canadian economy are immense. In the past decade, great advances have been made in understanding the genetic predisposition and biology of several polygenic disorders relevant to the mandate of INMD including Crohn's disease, ulcerative colitis, diabetes, polycystic kidney diseases and disorders of lipid metabolism. However, there remain critical gaps in knowledge related to the impact of genetic modifiers and local microenvironments on disease phenotype, clinical course of disease and variability in disease expression in genetically susceptible individuals. Seeking answers to such research questions has the potential to reduce the burden of illness in both common and rare diseases, to improve the quality of life of affected patients and to reduce health care inequities for populations of increased vulnerability.

Research advances that improve current understanding of the biology of rarely occurring human diseases and optimizing the care of such affected individuals have tremendous positive implications for quality of life. Past experience also clearly shows that such targeted research often has a much wider impact that influences the health of the population at large. For example, research defining the genetic basis of the rare condition Tangier's disease provided new insights relevant to lipid metabolism. This insight subsequently directed 
research aimed at providing novel interventions to prevent atherogenesis and its complications (cardiovascular disease and stroke) in the population at large.

Consulting with INMD stakeholders from across Canada revealed multiple examples of how this priority resonated. For example, INMD can enhance the knowledge base of natural environments and built environments relating to the pathobiology of both common and rare chronic diseases; support research evaluating the role of environments on chronic disease (eg, inflammatory bowel diseases and metabolic syndrome); and advance research on rare diseases, because this approach benefits the health of all Canadians.

\section{Strategic priority 3: Continuum of care}

INMD aims to improve the health care experience and health of people with chronic disease by fostering research on access to appropriate care including prevention and treatment. We will support research on new approaches to chronic disease prevention and management (eg, coordination of primary and specialty health care sectors), focus on transitions across different dimensions of the care and age continuum, and advance research that includes health care reform, care gaps and priority populations.

Rationale: The continuum of care may be defined as the array of health services that span the range over the life course from primary care (including prevention) through institutionally based secondary and tertiary care, to community and home-based services that promote health maintenance, rehabilitation and palliation at the end of life (4). INMD will support knowledge creation with respect to this priority by developing new approaches to prevent and manage chronic diseases with emphasis on diabetes, kidney and digestive diseases; optimizing coordination between specialists and primary care providers; and reducing care gaps across the continuum of care and the lifespan.

Canada's health care system faces critical challenges including capacity limitations related to Canada's aging population and an increasing prevalence of chronic disease. These challenges are extremely relevant to the INMD mandate in the areas of diabetes, kidney and digestive diseases, and will align with CIHR's new Strategy for Patient-Oriented Research.

Rising obesity rates, sedentary lifestyles, an aging population and changes in the ethnic mix of new immigrants will continue to drive diabetes rates in Canada upward. The economic burden of diabetes in Canada was estimated at approximately $\$ 12.2$ billion in 2010 , measured in inflation-adjusted 2005 dollars. The direct cost of diabetes now accounts for approximately $3.5 \%$ of public health care spending in Canada, and this share will continue to rise given the expected increase in the number of people with diabetes in Canada. People with diabetes incur medical costs that are up to three times higher than those without diabetes (5). Furthermore, the diabetes rates in Aboriginal people are up to five times higher than in non-Aboriginal populations (6).

Investments need to be made in research related to appropriate and timely access to health services, education, supplies and devices that will assist those with diabetes to manage their disease most effectively. While there is a genetic predisposition for diabetes, it is estimated that more than $50 \%$ of type 2 diabetes cases could be delayed - or even prevented - with healthier eating and increased physical activity (7).

Although fewer than $0.1 \%$ of Canadians have end-stage renal disease (ESRD), the disease generated direct health care costs of $\$ 1.3$ billion in the year 2000. According to the Canadian Institute for Health Information, the number of newly diagnosed ESRD (kidney failure) patients with diabetes increased by $114 \%$ over 10 years - from 1066 in 1995 to 2139 in 2004 (8). These figures highlight the need for innovative approaches to prevention, early detection and treatment of ESRD, as well as the long-term treatment of kidney disease.

The economic burden of illness associated with digestive diseases is also of considerable magnitude. Digestive diseases ranked fifth in terms of the direct economic costs of illness in Canada in 2000 (9). Direct costs include hospital care, drug expenditures, physician care expenses, expenditures for care in other institutions and additional health expenditures. Digestive disorders include irritable bowel syndrome, ulcers and colorectal cancer, which have a severe impact on quality of life and drive up health care costs. It is estimated that $42 \%$ of digestive diseases, including colorectal cancer, are preventable; however, there are gaps in health services that require attention (10).

\section{Strategic priority 4: Obesity and healthy body weight - seeking solutions}

INMD aims to support research on solution-focused interventions related to obesity at the clinical, policy and population health level. We will foster research on priority populations (eg, children, Aboriginal peoples and morbidly obese individuals), and emphasize knowledge translation to improve prevention approaches and enhance weight management strategies.

Rationale: Obesity continues to be a pressing public health challenge in Canada. In 2004, nearly one-quarter of adult Canadians (5.5 million people 18 years of age and older) were considered to be obese, and an additional $36 \%$ ( 8.6 million) were considered to be overweight (11). Evidence from the United States (12) indicates that "if past obesity trends continue unchecked, the negative effects on the health of the U.S. population will increasingly outweigh the positive effects gained from declining smoking rates. Failure to address continued increases in obesity could result in an erosion of the pattern of steady gains in health observed since the early 20th century".

This priority builds on INMD's previous strategic plan and CIHR investments. INMD commissioned a bibliometric study of obesity research in Canada (13) and found that, between 1998 and 2007, the number of obesity-related publications increased 2.6-fold; the number of Canadian obesity-related publications increased 3.7-fold; and the number of obesity-related publications by CIHR funded researchers increased 4.4-fold.

Increased capacity in the Canadian obesity research community correlates with an eight-fold increase in peer-reviewed funding supported by CIHR. Given the relative strength and size of the Canadian basic biomedical research community, the majority of these funds have supported research related to understanding the causal mechanism and consequences of obesity. INMD recognizes that there are still important basic research questions that need to be resolved. These types of proposals are well-suited to the CIHR open-grant competitions. CIHR has always provided, and will continue to provide, strong support for discovery-based research.

According to 2006 Canadian clinical practice guidelines on the management and prevention of obesity in children and adults (14), the level and grade of evidence for various interventions is very poor. Many of the recommendations for prevention had weak or no evidence. Practitioners, governments and the general public are all searching for effective ways to deal with overweight and obesity, and to promote healthy body weight. Additional research to delineate more efficient and effective strategies for preventing and treating obesity are needed. This is an area of research that INMD can support to have a positive impact on the health of Canadians.

INMD constituents overwhelmingly advised to continue supporting solution-focused research at both the individual and population levels. Among governments, nongovernmental organizations and health care professionals, there is a growing consensus about the need for additional research to help children maintain healthy body weights, to seek culturally relevant approaches for the Aboriginal population and to develop strategies to more effectively address the health care needs of those with morbid obesity. Public health agencies, governments and nongovernmental organizations are initiating large scale projects to address both the general population and specific priority populations.

INMD will support knowledge creation with respect to this research priority by focusing on solutions to promote healthy body weight at the population level (eg, school-based approaches and intervention research) and concentrating on interventions to prevent and 
manage obesity that align with CIHR's Patient-Oriented Research Strategy that evaluate the outcomes of specialized treatment services, provide a comparative analysis of clinical outcomes for bariatric surgical procedures and advance understanding of underlying mechanisms of action of various bariatric surgery options.

\section{CONCLUSIONS: MOVING FORWARD}

The present plan outlines the INMD strategic priorities for the next five years. The directions in this document will be used to guide future activities of the Institute, funding opportunities and knowledge translation initiatives. A detailed implementation and evaluation plan is being developed with the IAB. The CIHR mandate is to create new knowledge and translate this knowledge into improved health for Canadians, more effective health services and products and a strengthened Canadian health care system.

ACKNOWLEDGEMENTS: The authors thank Dr Brian Rush for his expertise as a facilitator in supervising the development of this strategic plan. The input and guidance of the Canadian health research community and of each member of the INMD Institute Advisory Board in formulating the content of this document are greatly appreciated. The INMD Strategic Plan is published by the CIHR as ISBN: 978-1-100-51533-5.

\section{REFERENCES}

1. WHO Technical Report Series 916, Diet, Nutrition and the Prevention of Chronic Diseases. Report of a Joint WHO/FAO Expert Consultation. WHO, 2003.

2. World Cancer Research Fund, American Institute for Cancer Research. Food, Nutrition, Physical Activity and the Prevention of Cancer: A Global Perspective. Washington DC: American Institute for Cancer Research, 2007.

3. Daar AS, Singer PA, Persad DL, et al. Grand challenges in chronic non-communicable diseases. Nature 2007:450:494-6.
4. Canadian Medical Association. CMA Policy: Funding the Continuum of Care. 2010. <http://policybase.cma.ca/dbtw-wpd/ Policypdf/PD10-02.pdf> (Accessed on July 15, 2011).

5. Canadian Diabetes Association. An Economic Tsunami: The Cost of Diabetes in Canada, December 2009. <www.diabetes.cal documents/get-involved/FINAL_Economic_Report.pdf> (Accessed on July 15, 2011).

6. Public Health Agency of Canada. National Diabetes Fact Sheet Canada 2008. <www.phac-aspc.gc.ca/publicat/2008/ndfs-fnrd-08/ ndfs_ff-fnrd_fc-eng.php> (Accessed on July 15, 2011).

7. Canadian Diabetes Association Clinical Practice Guidelines Expert Committee. Canadian Diabetes Association 2008 clinical practice guidelines for the prevention and management of diabetes in Canada. Can J Diabet 2008;32(Suppl 1):S1-S201.

8. Canadian Institute for Health Information, Treatment of End-Stage Organ Failure in Canada, 1995 to 2004 (2006 Annual Report). Ottawa: Canadian Institute for Health Information, 2006.

9. Public Health Agency of Canada. Economic Burden of Illness in Canada, 2000 (unpublished).

10. Canadian Digestive Health Foundation. Establishing Digestive Health as a Priority for Canadians. November 2009. <www.cdhf.ca/ pdfs/CDHF_National_Prevalence_Impact_Study_Report_ Nov_2009.pdf> (Accessed on July 15, 2011).

11. Tjepkema M, Shields M. Nutrition: Findings from the Canadian Community Health Survey, Issue no. 1. Measured Obesity: Adult Obesity in Canada. Component of the Statistics Canada Catalogue no 82-620-MWE2005001. <www.statcan.gc.ca/pub/82-620-m/82620-m2005001-eng.htm> (Accessed on July 15, 2011).

12. Stewart S, Cutler D, Rosen A. Forecasting the effects of obesity and smoking on U.S. life expectancy. N Engl J Med 2009;361:2252-60.

13. Girard G, Gendron JP, Macaluso B, Robitaille JP, Lariviere V. Observatoire des sciences et des technologies (OST). Bibliometric Study of Obesity Research in Canada 1998-2007, June 2009. $<$ www.cihr-irsc.gc.ca/e/41601.html > (Accessed on July 15, 2011)

14. Lau DC, Douketis JD, Morrison KM, Hramiak IM, Sharma AM, Ur E. 2006 Canadian clinical practice guidelines on the management and prevention of obesity in adults and children. CMAJ 2007;10;176:S1-13. 


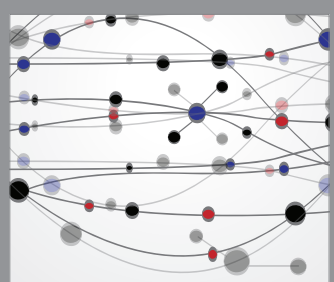

The Scientific World Journal
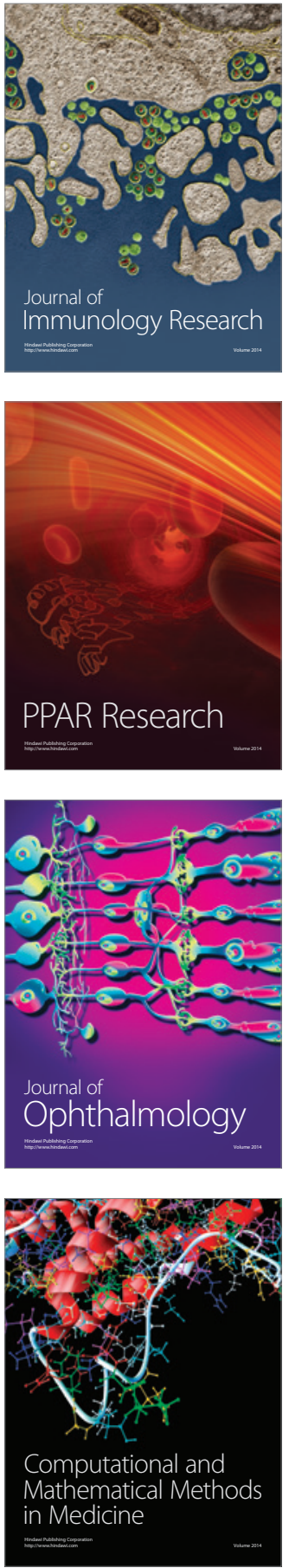

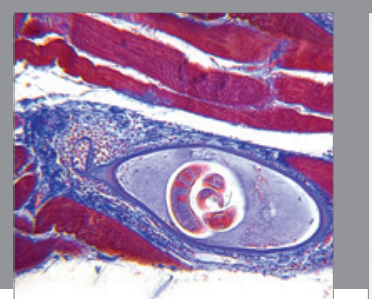

Gastroenterology Research and Practice

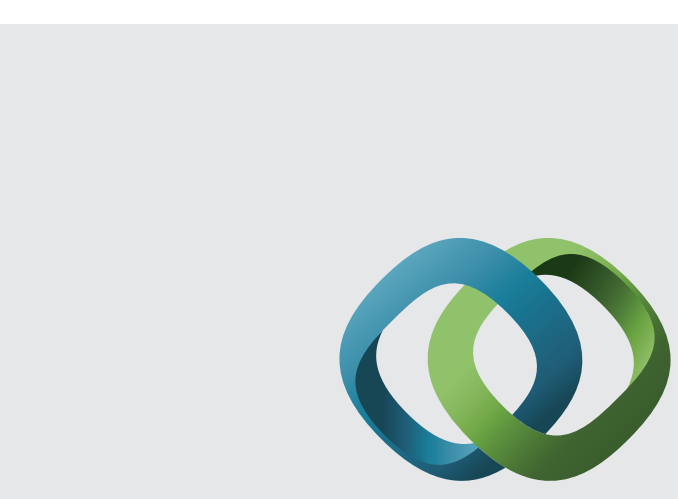

\section{Hindawi}

Submit your manuscripts at

http://www.hindawi.com
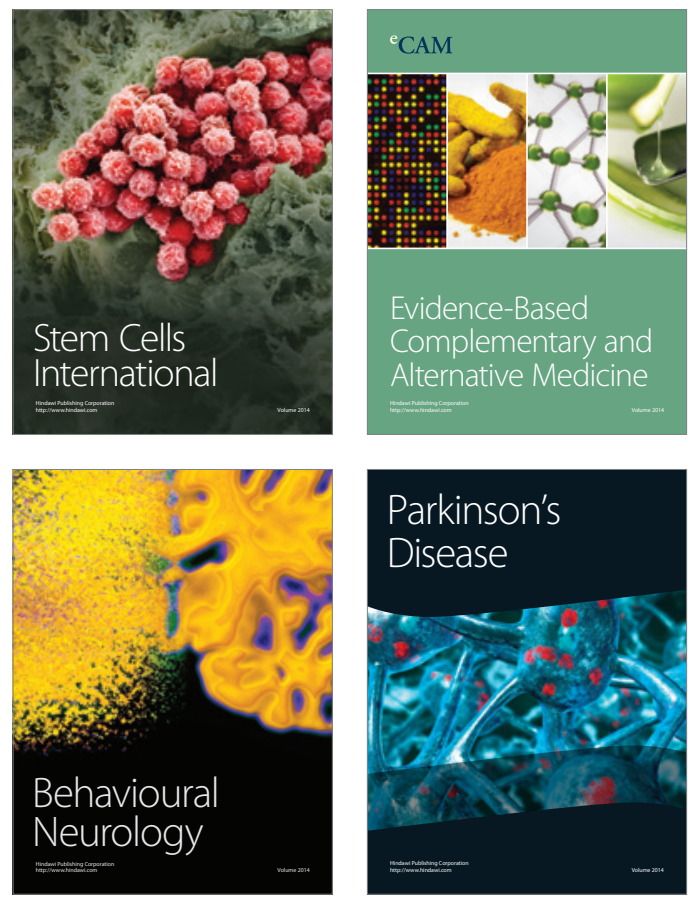
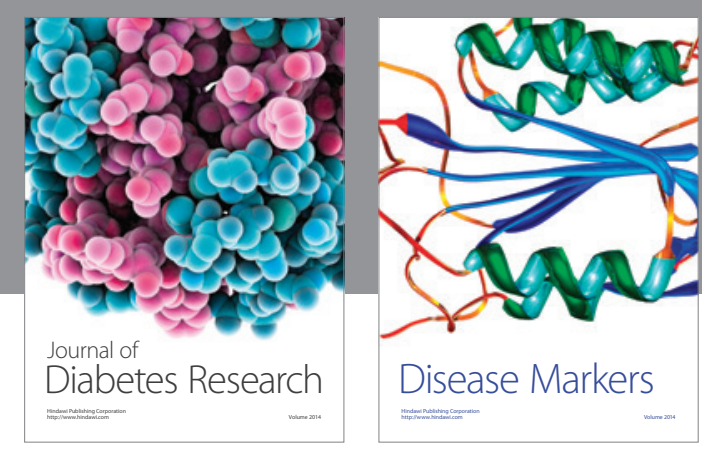

Disease Markers
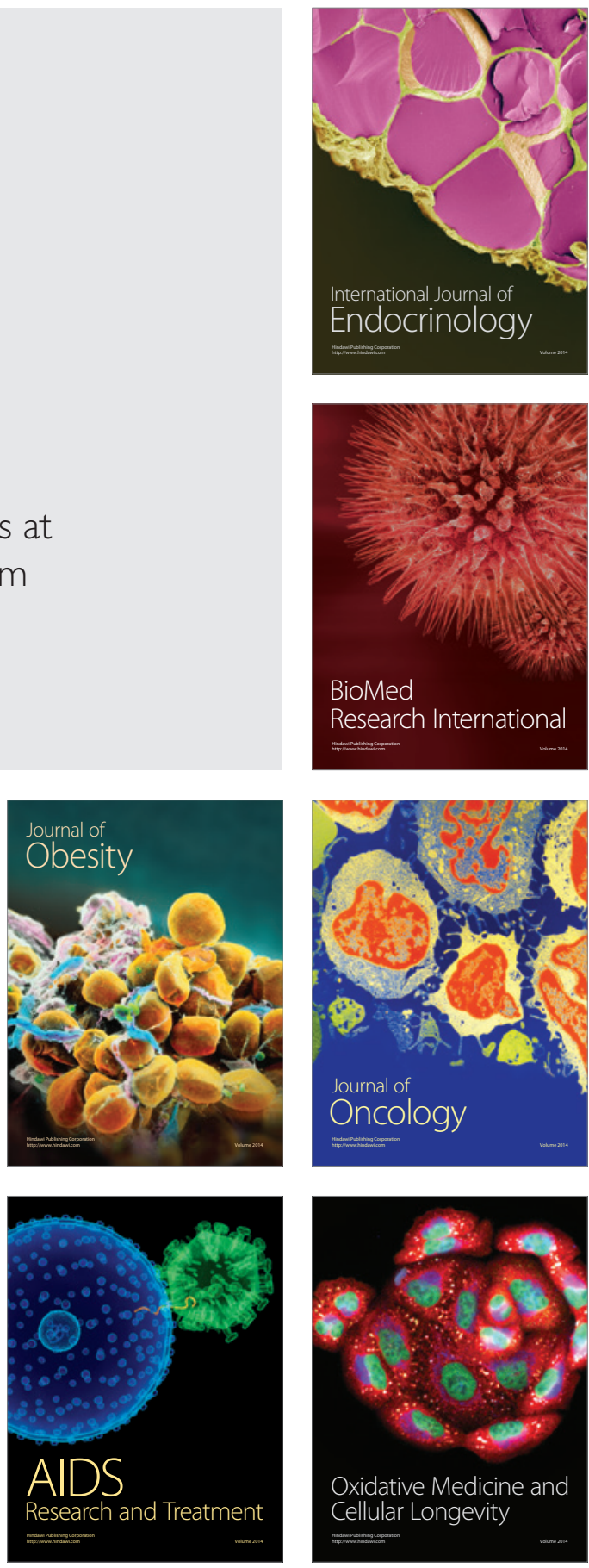